\title{
The Effects of a Novel Inhibitor of Tumor Necrosis Factor (TNF) Alpha on Prepulse Inhibition and Microglial Activation in Two Distinct Rodent Models of Schizophrenia
}

\section{Heath W. Shelton}

East Tennessee State University

Somasundar Prasad Gabbita

P2D Bioscience, Inc.

\section{W. Drew Gill}

East Tennessee State University

Katherine C. Burgess

East Tennessee State University

Wyatt S. Whicker

East Tennessee State University

Russell W. Brown ( $\square$ brown1@etsu.edu )

East Tennessee State University James H Quillen College of Medicine

\section{Research}

Keywords: Tumor Necrosis Factor-Alpha (TNFa), schizophrenia, neuroinflammation, Poly I:C, Dopamine D2 receptor, microglia, prepulse inhibition, sensorimotor gating

Posted Date: December 11th, 2019

DOl: https://doi.org/10.21203/rs.2.18552/v1

License: (1) This work is licensed under a Creative Commons Attribution 4.0 International License.

Read Full License 


\section{Abstract}

Background: Increased neuroinflammation has been shown in individuals diagnosed with schizophrenia (SCHZ). This study evaluated a novel immune modulator (PD2024) that targets the pro-inflammatory cytokine tumor necrosis factor-alpha (TNFa) to alleviate sensorimotor gating deficits and microglial activation employing two different rodent models of $\mathrm{SCHZ}$.

Methods: In Experiment 1, rats were neonatally treated with saline or the dopamine D2-like agonist quinpirole (NQ; $1 \mathrm{mg} / \mathrm{kg}$ ) from postnatal day $(\mathrm{P})$ 1-21 which produces increases of dopamine $\mathrm{D} 2$ receptor sensitivity throughout the animal's lifetime. In Experiment 2, rats were neonatally treated with saline or the immune system stimulant polyinosinic:polycytidylic acid (Poly I:C) from P5-7. Neonatal Poly I:C treatment mimics immune system activation associated with $\mathrm{SCHZ}$. In both experiments, rats were raised to $\mathrm{P} 30$ and administered a control diet or a novel TNFa inhibitor PD2024 $(10 \mathrm{mg} / \mathrm{kg})$ in the diet from P30 until P67. At P45-46 and from P60-67, animals were behaviorally tested on auditory sensorimotor gating as measured through prepulse inhibition (PPI).

Results: NQ or Poly I:C treatment resulted in PPI deficits, and PD2024 treatment alleviated PPI deficits in both models. Results also revealed that hippocampal microglial activation produced by neonatal Poly I:C was significantly reduced to control levels by PD2024. In addition, a separate group of animals neonatally treated with saline or Poly I:C from P5-7 demonstrated increased TNFa protein levels in the hippocampus, verifying increased TNFa in the brain produced by Poly I:C.

Conclusions: Results from this study suggests that that brain TNFa is a viable pharmacological target to treat the neuroinflammation known to be associated with $\mathrm{SCHZ}$.

\section{Introduction}

Individuals diagnosed with schizophrenia ( $\mathrm{SCHZ}$ ) have been shown to have increased inflammation in the brain CNS, particularly within the amygdala, hippocampus, nucleus accumbens, and prefrontal cortex (13). Inflammation is the first reaction of the body's immune system, which is a complex response of the host to tissue injury such as infection or physical insult (4). Prenatal maternal infections with pathogens such as herpes simplex, Toxoplasma gondii, or cytomegalovirus are associated with SCHZ in adult offspring (5-7). Further, autoimmune diseases in children or early childhood infections have been associated with higher incidence of $\operatorname{SCHZ}(8,9)$. The ensuing inflammation damages key components in the brain involved in neuronal plasticity, and ultimately contribute to cytokine and neurotransmitter abnormalities. It is hypothesized that changes in the immune system are a driving factor behind both psychotic relapses and the macroscopic brain changes that occur in $\mathrm{SCHZ}$, including the characteristic enlarged ventricle size and reductions in gray matter volume, whole-brain volume, as well as abnormalities in white matter (10).

Pro-inflammatory cytokines are recruited from the periphery in elevated levels in response to infection or insult that result in inflammation. Inflammation may interrupt normal processes in the blood brain barrier 
(BBB), which may contribute to the unusual peripheral recruitment pattern (11). Therefore, it becomes essential to further understand and investigate this inflammatory response in individuals diagnosed with $\mathrm{SCHZ}$ to potentially serve as a new drug target. In the abnormal inflammatory response for patients diagnosed with $\mathrm{SCHZ}$, increases of tumor necrosis factor-alpha (TNFa) levels, a pro-inflammatory cytokine, have been shown to be increased in the periphery and in the CNS $(12,13)$. TNFa is found in leukocytes and is involved in the acute phase reaction of inflammation. It is responsible for the regulation of a variety of immune cells that respond to inflammatory signals. Elevated levels of TNFa directly influence the state of microglia cells. Microglia are the primary immune cells of the CNS and constantly survey their local environment for signs of cellular stress (14). Under normal conditions, microglia exist as anti-inflammatory, neuroprotective agents, characterized as the "M2 state." However, upon activation by TNFa secretion, microglia switch to the "M1 state," which is pro-inflammatory and neurotoxic. Microglia in the $\mathrm{M} 1$ activation state lead to overexpression of pro-inflammatory cytokines and reactive oxygen species (ROS), resulting in synaptic loss and neuronal death (3).

Although targeting neuroinflammation and the development of new anti-inflammatories has become a novel therapeutic approach to treat $\mathrm{SCHZ}$, an increase of dopamine D2 receptor sensitivity still remains as a central hallmark of the disease. Our laboratory has established a rodent model of SCHZ based on increases in dopamine D2 receptor sensitivity through neonatal treatment of the dopamine D2-like agonist quinpirole to rats (15). The neonatal quinpirole rat model shows behavioral, neurobiological, and genetic consistencies that mimic features of individuals diagnosed with SCHZ (16). However, it is not yet known whether the neonatal quinpirole rat model displays neuroinflammatory features observed in human $\mathrm{SCHZ}$.

In the current study, we will evaluate the effects of a novel, orally bioavailable TNFa modulator, on auditory sensorimotor gating as measured through prepulse inhibition (PPI) in two rodent models of SCHZ: The neonatal quinpirole model and the polyinsinic:polycytidylic acid (Poly I:C) model. The rationale for employing two models that mimic different aspects of the disease is to perform a more rigorous and thorough evaluation of the TNFa modulator. The neonatal quinpirole model mimics the increases of dopamine D2 receptor sensitivity present in $\mathrm{SCHZ}$, whereas the Poly I:C model mimics neuroinflammation and the increase in immune response during early brain development that has been shown to increase the incidence of SCHZ. PPI is a behavioral task that involves the presentation of an auditory prepulse $(73,76$, or $82 \mathrm{~dB}$ ) presented shortly $(100 \mathrm{~ms})$ before a startling auditory stimulus (120 dB). Therefore, the prepulse predicts the startling auditory stimulus, and over presentations of these stimuli the animal acquires the ability to inhibit the startle response. Deficits in PPI are a behavioral hallmark of SCHZ. In addition, we will analyze the effects of a novel TNFa inhibitor on microglial activation in brain areas known to be critical in the circuitry that underlies PPI. Past work has shown that neonatal poly I:C treatment to rats increases proinflammatory cytokines and increases microglial activation in the hippocampus and prefrontal cortex $(17,18)$

\section{Methods}

Subjects. 
A total of 78 male Sprague-Dawley rats that were the offspring of 15 female Sprague-Dawley dams were subjects across both experiments. Females were not included in this study because of past work that has reported equivocal results relative to sex differences on the auditory sensorimotor gating task in different models of SCHZ and/or neuroinflammation $(19,20)$. One rat from each litter were assigned to each drug/diet condition to control for within litter variance. The day of birth was counted as postnatal day (P)0. Regardless of neonatal drug treatment, all animals were housed with the female dam from P121 and socially housed from P22-30. Beginning on P30, the control or experimental diet was presented, and all animals were singly housed from P30 until the P67, when brain tissue was harvested. Animals were housed in a climate-controlled vivarium with a $12 \mathrm{~h}$ on/off light/dark cycle throughout the experiment. Procedures used in this study were approved by the University Committee on Animal Care at East Tennessee State University and animal facilities are accredited by the American Association of Laboratory Animal Care (AAALAC).

PD2024.

PD2024 is part of an isoindoline-based drug technology that P2D Bioscience, Inc (Cincinnati, OH) licensed from the $\mathrm{NIH}$ and is being developed to treat dementia. Past work from Gabbita and colleagues reported that oral PD2024 engaged brain TNFa, improved cognitive performance, and reduced the core phosphotau tangle immune-histopathology in a mouse model of Alzheimer's Disease (21).

\section{Experiment 1: Drug/Diet Treatment.}

In Experiment 1, rats were neonatally treated with saline or the dopamine D2-like agonist quinpirole. A total of 24 rats were neonatally i.p. administered saline or quinpirole $(1 \mathrm{mg} / \mathrm{kg})$ from postnatal day (P)1-21, identical to past work with this model (see 16 for review). Our lab and collaborators have shown that neonatal quinpirole (NQ) treatment from P1-21 produces increases in dopamine D2 receptor sensitivity throughout the animal's lifetime (Kostrzewa, et al, 1995).

\section{Experiment 2a: Drug/Diet Treatment.}

In Experiment 2, rats were neonatally i.p. administered either saline or Polyinosinic:Polycytidylic acid (Poly I:C) from P5-7, which has been shown to result in deficits in auditory sensorimotor gating as measured by PPI as well as increased microglial activation (18). In both experiments, beginning at P30, rats were singly housed with enrichment and given either regular chow or $10 \mathrm{mg} / \mathrm{kg}$ PD2024 (P2D Bioscience, Inc., Cincinnati, $\mathrm{OH}$ ) mixed in their diet (Dyets, Inc., Bethlehem, PA). These diets were presented until the end of the experiment when brain tissue was harvested at P67. The rationale for the use of this dose of PD2024 is that Gabbita and colleagues have shown that this dose, given through the diet, was effective to reduce inflammation as measured by microglial activation in a mouse model of Alzheimer's Disease (3xTg; 21).

Experiment $2 b$. 
Finally, in Experiment 2b, a separate group of 17 male Sprague-Dawley rats were administered saline $(\mathrm{N}=8)$ or Poly I:C ( $N=9)$ from P5-7, and raised to P30. These animals were not given any diet manipulation. For this group of animals, brain tissue was harvested at P30-33 and the hippocampus and prefrontal cortex were dissected away and flash frozen on dry ice. The brain tissue from these animals was analyzed for TNFa levels using an ELISA kit (Biomatik, Wilmington, DE) to verify that TNFa was increased by neonatal Poly I:C treatment when the diet manipulation was initiated.

\section{Group codes.}

With each neonatal drug treatment manipulation, there were four different conditions. In Experiment 1 the treatment groups were noted as NS PD2024, NQ PD2024, NS Control, NQ Control, with the first acronym representing neonatal drug treatment (NS-Neonatal Saline; NQ-Neonatal Quinpirole) and the second term representing which diet the group received beginning on P30. Likewise, in animals treated with Poly I:C, there were also four different conditions that were noted as NS PD2024, Poly IC PD2024, NS Control, and Poly IC Control with the first acronym representing neonatal drug treatment (NS-Neonatal Saline; Poly IC) and the second term representing which diet the group received beginning on P30.

\section{Experiments 1 \& 2: PPI Methods.}

Animals were behaviorally tested once daily on PPI and at two different time points: once during development adolescent days P45 and 46, and again in adulthood (P60-65). We opted to use two different developmental time points to analyze the effects of PD2024 over a developmental time course. The equipment used for PPI behavioral testing was from Kinder Scientific, Inc., (Poway, CA, USA). Rats were administered three different, randomly assigned trial types, which included pulse trials, prepulse trials, and no stimulus trials. A pulse trial is a $120 \mathrm{~dB}$ startle pulse administered alone. A prepulse trial is an auditory stimulus (click) that is 3,6 , or $12 \mathrm{~dB}$ above the $70 \mathrm{~dB}$ background noise (i.e. 73,76 , and $82 \mathrm{~dB}$ ). A no stimulus trial is a trial in which no stimulus is given. All animals underwent 25 randomized trials which include 5 pulse, 5 no stimulus, and 15 prepulse trials ( 5 trials of each 73,76 , and $82 \mathrm{db}$ ). Animals were placed into a stainless-steel dome (height $=8 \mathrm{~cm}$ ) that was attached to a platform $(11 \mathrm{~cm}$ wide $\times 15 \mathrm{~cm}$ long) mounted on a stainless steel ellipse in a sound attenuating chamber $(28 \mathrm{~cm}$ tall $\times 30 \mathrm{~cm}$ wide $\times 36$ $\mathrm{cm}$ in depth). The behavioral response was recorded during a $250 \mathrm{~ms}$ window immediately after the stimulus was presented, and was measured in Newtons, the SI (International System of Units) unit for force. On each daily session, the animals were placed into the dome and given a 5 min habituation period with only the background noise (70 dB white noise) presented.

Experiment 2a: Immunohistochemistry (IHC) for microglial activation in the hippocampus and prefrontal cortex.

We performed IHC only on animals neonatally treated with Poly I:C (Experiment 2), because it is well established that Poly I:C administered during either gestation (23) or neonatal development results in increases of microglial activation in the brain (Ribeiro et al 2013). On P67-69, Poly I:C animals were deeply anesthetized and intracardially perfused with $4 \%$ paraformaldehyde. Brain tissue was removed and stored 
in $20 \%$ sucrose for approximately $48 \mathrm{~h}$, and then transferred to a clean vial and stored at $-80^{\circ} \mathrm{C}$. Tissues were coronally sectioned on a Leica cryostat at $50 \mu \mathrm{m}$ thickness and stored at $-20^{\circ} \mathrm{C}$ until immunolabeling was performed. We chose to analyze the hippocampus and prefrontal cortex because their established roles in sensorimotor gating (24).

Free floating sections were mounted on glass slides and coverslipped using mounting media. Iba1 (Wako Chemicals USA, Richmond, VA) was the primary antibody used for IHC labeling of microglial cells and AlexaFluor488 conjugated Anti-Rabbit IgG (Jackson ImmunoResearch Laboratories, West Grove, PA) was the secondary antibody tagged with GFP to emit fluorescence. Slides were examined under a Leica TCS SP8 inverted confocal microscope at a magnification of 40x. A total of four images were captured per brain region (4 PFC, 2 CA1 \& 2 CA3 of the Hip). NIH ImageJ software was used to quantify images of the prefrontal cortex and CA1 and CA3 regions of the dorsal hippocampus. For each brain section, average sampled cell body fluorescence was measured. The freehand tool in ImageJ was used to draw around a given microglia cell body. Integrated Density from ImageJ was used to determine average cell body fluorescence as well as overall field fluorescence. At random, five GFP-stained microglia cells were selected per given field for the measurement of average cell body fluorescence.

\section{Experiment 2b: TNFa protein assay (TNFa ELISA).}

Animals neonatally treated with saline or Poly IC (2 mg/kg) brain tissue was taken between P30-33. This time point was chosen because this is when the dietary manipulation was begun. Once tissue was harvested, the dorsal hippocampus, ventral hippocampus, and medial frontal cortex were dissected away. The procedures for the kit obtained from Biomatik, Inc. (Wilmington, DE) were strictly followed with a few exceptions. In brief, $500 \mu$ l of RIPA cell lysis buffer (150 mM NaCL, 50 mM Tris-HCl, 1.0\% NP-40, $0.5 \%$ Sodium deoxycholate and 0.1\% SDS) plus protease and phosphatase inhibitors (P5726, P8340, P0044, Sigma-Aldrich, St. Louis, MO) was added to each tissue sample and homogenized using a Fisher Scientific sonic dismembrator 500 (Fisher Scientific, Inc, Atlanta, GA). Homogenates were then centrifuged at $10,000 \mathrm{~g}$ for $5 \mathrm{~min}$ at $4^{\circ} \mathrm{C}$. For the TNFa assay, the 96 well plate arrived coated with anti-TNFa polyclonal antibody (mAb) to each well of a 96-well polystyrene ELISA plate provided in the kit. The TNFa standard curve was prepared using the TNFa standard supplied form the manufacturer $(10,000 \mathrm{pg} / \mathrm{ml})$. The standard was diluted by diluent supplied by the kit to achieve a concentration range of 15.6-1,000 pg/ml. Tissue samples were further diluted 1:50 before being assayed. The standards and samples were incubated at room temperature for 1 hour. The monoclonal antibody was then added to each well plate, incubated at room temperature $(1 \mathrm{~h})$, which was followed by incubation $(1 \mathrm{~h})$ with the conjugate. Visualization was achieved by adding tetramethybenzidine (TMB) one solution to each well followed by an incubation period of 20 minutes at room temperature, and this reaction was stopped by adding the stop solution to each well and plates were read within 5 minutes of stopping the reaction. Optical density was measured using a Bio-Tek ELx 800 microplate reader (Winooski, VT).

Statistical Analysis. 
For weight gain analysis in each experiment, a three-way ANOVA was used with neonatal drug treatment, diet (control or PD2024), and day of development (P30, P40, P50, P60) was used as the repeated measure. For analysis of PPI, the two experiments were separately analyzed. In each experiment, a three-way ANOVA was used with neonatal drug treatment, diet (control or PD2024), and prepulse $(73,76$, or $82 \mathrm{~dB}$ ) as between subjects factors. A two-way ANOVA (neonatal drug treatment, diet) was used to analyze IHC immunofluorescence in each brain area. The Newman-Keuls post hoc test $(p=0.05)$ was used to analyze any significant interactions in both experiments.

\section{Results}

Weight Gain. The weight of animals across neonatal drug treatments and diet from P30-60 are presented in Table 1 for Experiment 1 and 2. A three-way ANOVA revealed no significant main effects or interactions. Therefore, regardless of neonatal drug treatment and diet condition, there was no significant main effects of interactions of the PD2024 diet on normal weight gain in each neonatal drug treatment condition.

Table 1a. Animal's body weight for Experiment 1.

Means for each drug treatment group and day of development are presented with standard errors of the mean in parentheses.

\begin{tabular}{|l|l|l|l|l|l|}
\hline $\begin{array}{l}\text { Neonatal Drug } \\
\text { Treatment }\end{array}$ & Diet & $\begin{array}{l}\text { P30 } \\
\text { Weight }\end{array}$ & P40 Weight & P50 Weight & P60 Weight \\
\hline Saline & Control & $86.8(4.57)$ & $162.2(6.5)$ & $231.7(6.96)$ & $289(9.2)$ \\
\hline Quinpirole & Control & $89.8(3.01)$ & $166(5.1)$ & $235.8(6.43)$ & $297.8(6.9)$ \\
\hline Saline & PD2024 & $87(4.46)$ & $\begin{array}{l}158.3 \\
(6.55)\end{array}$ & $223(7.45)$ & $281.3(8.6)$ \\
\hline Quinpirole & PD2024 & $94.5(2.75)$ & $\begin{array}{l}169.2 \\
(3.51)\end{array}$ & $239(4.22)$ & $300(6.5)$ \\
\hline
\end{tabular}

Table 2a. Animal's body weight for Experiment 2.

Means for each drug treatment group and day of development are presented with standard errors of the mean in parentheses. 


\begin{tabular}{|l|l|l|l|l|l|}
\hline $\begin{array}{l}\text { Neonatal Drug } \\
\text { Treatment }\end{array}$ & Diet & P30 Weight & P40 Weight & P50 Weight & P60 Weight \\
\hline Saline & Control & $87.7(6.30)$ & $\begin{array}{l}159.0 \\
(7.67)\end{array}$ & $\begin{array}{l}223.4 \\
(5.21)\end{array}$ & $278.3(7.2)$ \\
\hline Poly IC & Control & $98.9(2.29)$ & $170.3(4.1)$ & $\begin{array}{l}244.0 \\
(4.25)\end{array}$ & $304.1(5.7)$ \\
\hline Saline & PD2024 & $100.3(4.6)$ & $165.7(5.6)$ & $226.0(5.1)$ & $278.3(7.1)$ \\
\hline Poly IC & PD2024 & $\begin{array}{l}100.3 \\
(2.65)\end{array}$ & $\begin{array}{l}168.4 \\
(6.14)\end{array}$ & $240.0(9.8)$ & $\begin{array}{l}294.4 \\
(12.8)\end{array}$ \\
\hline
\end{tabular}

Experiment 1: PPI in NQ/NS-treated Adolescent rats (P45/46).

PPI results for NQ/NS-treated adolescent rats are in Figure 1a. A three-way ANOVA revealed a significant two-way interaction of neonatal drug treatment $\mathrm{x} \operatorname{diet} \mathrm{F}(1,20)=9.93$, $\mathrm{p}<.005$. Results revealed that NQ-Controls and NS-PD2024 demonstrated a significant deficit to NS-Control and NQ-PD2024 at the 76 dB BS 82 dB intensity, but there were no significant group differences at the 73 or $82 \mathrm{~dB}$ intensity. Thus, NQ produced a significant PPI deficit at 76 and 82 dB prepulse intensities which was alleviated by PD2024. However, PD2024 produced a deficit in control animals at these same prepulse intensities. This is not necessarily unexpected because PD2024 is designed to modulate TNF $\alpha$ in an abnormal system which, in this case, resulted in deficits in PPI.

Experiment 1: PPI in NQ/NS-treated Adult rats (P60-65).

PPI results for NQ/NS-treated adult rats are in Figure 1b. A three-way ANOVA revealed a significant main effect of $\operatorname{diet} F(1,20)=15.19, \mathrm{p}<.001$, a significant two-way interaction of neonatal drug treatment $\mathrm{x} \operatorname{diet} \mathrm{F}(1,20)=22.69, \mathrm{p}<.001$, and a significant threeway interaction of neonatal $x$ diet $x$ prepulse intensity $F(2,40)=6.29, p<.004$. In sum, NQcontrol rats demonstrated a significant deficit at all three prepulse intensities. Unlike adolescents, PD2024 did not result in a deficit in NS-treated animals.

Experiment 2a: PPI in Poly IC/NS-treated Adolescent rats (P45-46). 
PPI results for Poly I:C/NS-treated adolescent rats are in Figure 2a. A three-way ANOVA revealed a significant two-way interaction of neonatal drug treatment $\mathrm{x}$ diet $\mathrm{F}(1,32)$ $=16.72, \mathrm{p}<.001$. Across all prepulse dB intensities, NS Control and Poly I:C-PD2024 were equivalent, and demonstrated significantly improved PPI performance compared to Poly I:CControls and NS-PD2024 groups. NS-Controls and the Poly IC-PD2024 group were statistically equivalent across all decibel levels.

Experiment 2a: PPI in Poly IC/NS-treated Adult rats (P60-65).

PPI results for Poly I:C/NS-treated adult rats are in Figure 2a. A three-way ANOVA revealed a significant main effect of $\operatorname{diet} \mathrm{F}(1,32)=8.8, \mathrm{p}<.006$ and a significant interaction of neonatal drug treatment $\mathrm{x}$ diet $(\mathrm{F} 1,32)=23.01, \mathrm{p}<.001$. Similar to the results in adulthood with the NQ model, PD2024 alleviated PPI deficits produced by neonatal poly I:C treatment and deficits in PPI observed in NS-treated adolescent rats treated with PD2024 dissipated in adulthood. Improvement in PPI deficits in both models ranged between 15 to $30 \%$ in adolescence and adulthood.

Experiment 2a: Microglial action in Poly IC-treated rats (P66-69).

Microglial results for rats neonatally treated with saline or Poly I:C are presented in Figure 3. A separate one-way ANOVA was used to analyze each brain area, and revealed a significant main effect of group in the prefrontal cortex $F(3,15)=8.0, p<.003$, hippocampal area CA1 $\mathrm{F}(3,16)=8.1, \mathrm{p}<.003$, and hippocampal area CA3 $\mathrm{F}(3,16)=12.8, \mathrm{p}<.001$. Regardless of the brain area, rats administered poly I:C during the neonatal period demonstrated a significant increase in fluorescence intensity in microglia which was reduced to saline control levels by PD2024. These data indicate that PD2024 was effective to reduce the increase inflammatory response within microglia across three brain areas that are important in both working memory and auditory sensorimotor gating.

Experiment 2b. TNF $\alpha$ protein levels in Poly I:C-treated rats (P33-35).

$\mathrm{TNF} \alpha$ results for adolescent rats neonatally treated with saline or poly I:C are presented in Figure 4. An independent t-test was performed to analyze each brain area, and 
revealed a significant main effect of group in the dorsal hippocampus $t(12)=2.21, p<.05$ as well as the ventral hippocampus $\mathrm{t}(12)=3.16, \mathrm{p}<.01$. Although poly I:C increased absolute TNF $\alpha$ levels in the frontal cortex, it was not statistically significant due to high variability in the poly I:C-treated group ( $\mathrm{N}=5$ for this brain area). These results are consistent with recent work (25) that recently demonstrated maternal poly I:C significantly increased TNF $\alpha$ in the brain, but did not affect other pro-inflammatory cytokines.

\section{Discussion}

The present study demonstrated that a modulator of the pro-inflammatory cytokine TNFa, PD2024, presented in the diet during adolescence and early adulthood successfully alleviated deficits in auditory sensorimotor gating as measured by PPI in rats neonatally treated with the dopamine D2-like agonist quinpirole or the immune stimulant poly I:C. These results demonstrate the utility of PD2024 to alleviate PPI deficits in two well-validated but distinct rodent models of $\mathrm{SCHZ}$ that are related to either increase in dopamine D2 receptor functioning or early immune system activation. These findings are consistent with several past studies that have shown that the anti-inflammatories are effective to treat SCHZ. Likely, the most impactful of these studies utilized minocycline, which also decreases TNFa expression (26).

Minocycline was successful in alleviating PPI deficits in the poly I:C rodent model of SCHZ $(23,27)$. Finally, it was established that neonatal Poly I:C results in a significant increase in TNFa in the hippocampus, consistent with a recent study by Clark et al (25) that demonstrated increases in TNFa protein, but no changes in other pro-inflammatory cytokines, in the brain after maternal (GD15) acute infusion of Poly I:C $(5 \mathrm{mg} / \mathrm{kg}$ ). Since the known target of PD2024 is to modulate TNFa, this would at least suggest that its action on this pro-inflammatory cytokine is involved in mediating the effects observed in the present study.

PD2024 resulted in PPI deficits in the control group in adolescent rats in the present study, however, this deficit dissipated in adulthood. The mechanism for this effect is unknown, but interestingly, it was consistent across both models, indicating that there may be an interaction of PD2024 with neurobiological changes occurring during the adolescent period. However, this is really not a major concern, because PD2024 is being developed as a treatment only for individuals diagnosed with SCHZ who possess an abnormal central nervous system.

PD2024 is part of a drug technology that P2D Bioscience licensed from the National Institutes of Health $(\mathrm{NIH})$ and is being developed to treat dementia-related cognitive dysfunction (Alzheimer's and frontotemporal dementia), as psychosis has now been associated with dementia disorders (Kolanowski et al 2017). Preclinical efficacy studies have shown that oral PD2024 destabilizes TNFa DNA, improves cognitive performance, and reduces the core phosphotau tangle immunohistopathology associated with a mouse model of Alzheimer's Disease (21). The present study demonstrates that PD2024 was effective to alleviate a behavioral deficit that is a hallmark of SCHZ: auditory sensorimotor gating deficits as tested by PPI (29), demonstrating PD2024 may have utility as a treatment for a disease state beyond Alzheimer's 
disease. Improvements in PPI deficits in SCHZ have been related to cognitive impairment known to exist in the disorder (30).

Regarding the Poly I:C model, there is ample evidence to suggest that exposure to a prenatal infection during the first or second trimester of pregnancy can disrupt neurodevelopment, increasing the risk of developing neuropsychiatric disorders such as $\mathrm{SCHZ}(31,32)$. We show here that neonatal poly I:C activates microglia, and increases in microglial activation is known to cause white matter injury and neuronal apoptosis $(33,34)$. Consequently, a number of behavioral deficits consistent with $\mathrm{SCHZ}$ have been shown in the poly l:C model, including cognitive deficits and deficits in sensorimotor gating $(35,36)$. Results here are consistent with past work, including work that has administered poly I:C neonatally (18), as we did here, as well as maternal exposure to poly I:C. Interestingly, maternal $(37,38)$ exposure to poly I:C has demonstrated dopamine hyperfunction, and structural abnormalities including reduced hippocampal and prefrontal cortical volumes in poly l:C offspring. All of these characteristics are hallmarks of SCHZ (3). An advantage of both of these models is that these alterations emerge in the offspring during adolescence, which is consistent with clinical observations. A critical observation in any model is that treatments that typically alleviate some of the behavioral abnormalities of $\mathrm{SCHZ}$ are also observed in the model. Importantly, clozapine (18) as well as risperidone (39) have been shown to alleviate behavioral deficits observed in rats developmentally treated with poly I:C.

We analyzed changes in microglia only in animals neonatally treated with poly $\mathrm{I}: \mathrm{C}$. The rationale for this analysis was that it was known that poly I:C results in microglial activation $(40,41)$. A primary focus of this study was to demonstrate that PD2024 would reduce this microglial activation. Poly I:C did in fact increase microglial activation which was reduced to control levels by PD2024. The increase in microglial activation produced by neonatal Poly I:C treatment is consistent with past work and was observed in both hippocampus and prefrontal cortex, whereas past work had observed this effect in hippocampus, prefrontal cortex, and striatum. Presumably, PD2024 reduced this activation through modulating TNFa, however, it is also known that PD2024 increases cholinergic activity in the brain (Gabbita, unpublished observations). Increased nicotinic acetylcholinergic receptor activity has been associated with antiinflammatory properties (42). Interestingly, it has been discovered that acute postnatal Poly l:C (5 mg/ $\mathrm{kg})$ administered on P5 reduced binding at muscarinic receptors, suggesting a decrease in cholinergic activity due to neonatal Poly I:C treatment. Cholinergic agonists, especially at the nicotinic alpha7 receptor, have been shown to reduce PPI deficits and cognitive impairments in individuals diagnosed with SCHZ $(43,44)$. Therefore, PD2024 could be working through multiple mechanisms to alleviate PPI deficits observed in the present study.

Recently, the involvement of the immune system has increasingly been implicated in the behavioral pathological processes of $\mathrm{SCHZ}(45-48)$. It has been hypothesized that changes in the immune system is a driving factor behind both psychotic relapses and the macroscopic brain changes that occur in $\mathrm{SCHZ}$, including the characteristic enlarged ventricle size and reductions in gray matter volume, whole-brain volume, as well as the known abnormalities in white matter (10). McEvoy et al. recently published an analysis of inflammatory markers analysis of blood samples from $\mathrm{SCHZ}$ patients enrolled in the Clinical 
Antipsychotic Trials in Intervention Effectiveness (CATIE) project, which were suggestive that inflammation is associated with SCHZ-related psychopathology and cognition and has differential effects of substance use in $\mathrm{SCHZ} \mathrm{(49).} \mathrm{Although} \mathrm{the} \mathrm{results} \mathrm{at} \mathrm{this} \mathrm{point} \mathrm{are} \mathrm{not} \mathrm{conclusive,} \mathrm{there} \mathrm{are} \mathrm{supporting} \mathrm{data} \mathrm{to}$ indicate that the anti-inflammatory minocycline improved PANSS scores relative to placebo (For review see, 50,51) and more recently meta-analyses published in 2017 from both Solmi et al 52) and Xiang et al (53) reported minocycline's superiority over placebo, especially on total and negative symptom scores.

Increases in dopamine D2 receptor sensitivity has long been established as a neurobiological abnormality in $\mathrm{SCHZ} \mathrm{(3).} \mathrm{Support} \mathrm{for} \mathrm{this} \mathrm{notion} \mathrm{comes} \mathrm{from} \mathrm{the} \mathrm{fact} \mathrm{that} \mathrm{all} \mathrm{antipsychotic} \mathrm{medications} \mathrm{antagonize} \mathrm{the}$ dopamine D2 receptor with some affinity, and all of antipsychotic drugs have been shown to alleviate, at the very least, the positive symptoms of $\mathrm{SCHZ}$ (54). Over the past 15 years, our laboratory has investigated behavioral and neurobiological mechanisms in the neonatal quinpirole model, many that are consistent with SCHZ (16). However, this is the first demonstration that a drug designed to modulate a proinflammatory cytokine was effective at alleviating behavioral deficits in this model. On the other hand, we have published several studies analyzing nicotine in this model because of the high incidence of cigarette smoking in the population diagnosed with $\mathrm{SCHZ}$ (55). As mentioned above, the known anti-inflammatory properties of nicotine may be playing a role in this model as well, although we have yet to verify there is neuroinflammation in the neonatal quinpirole model. Nicotine is known to alleviate behavioral impairments in $\mathrm{SCHZ}$, including cognitive deficits (56). Therefore, this may represent a mechanism by which nicotine is working in $\mathrm{SCHZ}$, since it has recently been established neuroinflammation occurs in $\mathrm{SCHZ}$.

\section{Conclusions}

This study revealed that PD2024, a novel compound, directed at modulating the pro-inflammatory cytokine TNFa was effective at alleviating deficits in auditory sensorimotor gating, a behavioral hallmark of SCHZ. In addition, this compound also reduced microglial activation in the hippocampus, a brain area that plays a critical role in the symptoms expressed in individuals diagnosed with $\mathrm{SCHZ}$. Future work is designed to evaluate the underlying molecular mechanism by which PD2024 lowers TNFa and advance the compound's use as a possible adjunctive therapy to treat $\mathrm{SCHZ}$.

\section{References}

1. Na KS, Jung HY, Kim YK. The role of pro-inflammatory cytokines in the neuroinflammation and neurogenesis of schizophrenia. Prog Neuropsychopharmacol Biol Psychiatry. 2014;48: 277-86.

2. Weickert CS, Weickert TW. What's hot in schizophrenia research? Psychiatr Clin North Am. 2016;39: 343-51.

3. Howes OD, McCutcheon R, Owen MJ, Murray RM. The role of genes, stress, and dopamine in the development of schizophrenia. Biol Psychiatry. 2017;81: 9-20. 
4. Nathan C. Points of control in inflammation. Nature. 2002;420: 846-52. 5. Schmitt A, LeonardiEssmann F, Durrenberger PF, Parlapani E, Schneider-Axmann T, Spanagel R, Arzberger T, Kretzschmar H, Herrera-Marschitz M, Gruber O,Reynolds R, Falkai P, Gebicke-Haerter PJ. Regulation of immune-modulatory genes in left superior temporal cortex of schizophrenia patients: a genome-wide microarray study. World $\mathrm{J}$ Biol Psychiatry. 2011;12: 201-15.

6. Pandey GN, Rizavi HS, Zhang H, Ren X. Abnormal gene and protein expression of inflammatory cytokines in the postmortem brain of schizophrenia Schizophr Res. 2018;192: 247-54.

7. Brown AS, Derkits EJ. Prenatal infection and schizophrenia: A review of epidemiologic and translational studies. Am J Psychiatry. 2010;167: 261-80.

8. Khandaker GM, Zimbron J, Dalman C, Lewis G, Jones PB. Childhood infection and adult schizophrenia: a meta-analysis of population-based studies. Schizophr Res. 2012;139: 161-68.

9. Khandaker GM, Zimbron J, Lewis G, Jones PB. Prenatal maternal infection, neurodevelopment and adult schizophrenia: a systematic review of population-based studies. Psychol Med. 2013;43: 23957.

10. Bakhshi K, Chance SA. The neuropathology of schizophrenia: a selective review of past studies and emerging themes in brain structure and cytoarchitecture. Neuroscience. 2015;303:82-102

11. Schroeter M, Dennin MA, Walberer M, Backes H, Neumaier B, Fink GR, Graf R. Neuroinflammation extends brain tissue at risk to vital peri-infarct tissue: a double tracer [11C]PK11195- and [18F]FDG-PET study. J Cereb Blood Flow Metab. 2009;29: 1216-25. 12. Miller BJ, Buckley P, Seabolt W, Mellor A, Kirkpatrick B. Meta-analysis of cytokine alterations in schizophrenia: clinical status and antipsychotic effects. Biol Psychiatry. 2011;70: 663-71. 13. McCusker RH, Kelley KW. Immune-neural connections: how the immune system's response to infectious agents influences behavior. J Exp Biol. 2013;216(Pt 1): 84-98. 14. Nimmerjahn A, Kirchhoff F, Helmchen F. Resting microglial cells are highly dynamic surveillants of brain parenchyma in vivo. Science. 2005 308:1314-8. 15. Kostrzewa RM, Wydra K, Filip M, Crawford CA, McDougall SA, Brown RW, Borroto-Escuela DO, Fuxe K, Gainetdinov RR. Dopamine D(2) receptor supersensitivity as a spectrum of neurotoxicity and status in psychiatric disorders. J Pharmacol Exp Ther. 2018;366: 519-26.

16. Brown RW, Maple AM, Perna MK, Sheppard AB, Cope ZA, Kostrzewa RM. Schizophrenia and substance abuse comorbidity: nicotine addiction and the neonatal quinpirole model. Dev Neurosci. 2012;34: 140-51.

17. Gandhi R, Hayley S, Gibb J, Merali Z, Anisman, H. Influence of poly I:C on sickness behaviors, plasma cytokines, corticosterone and central monoamine activity: moderation by social stressors. Brain Behav Immun. 2007;21: 477-89.

18. Ribeiro BM, do Carmo MR, Freire RS, Rocha NF, Borella VC, de Menezes AT, Monte AS, Gomes PX, de Sousa FC, Vale ML, de Lucena DF, Gama CS, Macêdo D. Evidences for a progressive microglial activation and increase in iNOS expression in rats submitted to a neurodevelopmental model of schizophrenia: reversal by clozapine. Schizophr Res. 2013;151: 12-9. 
19. Foley KA, MacFabe DF, Kavaliers M, Ossenkopp KP. Sexually dimorphic effects of prenatal exposure to lipopolysaccharide, and prenatal and postnatal exposure to propionic acid, on acoustic startle response and prepulse inhibition in adolescent rats: relevance to autism spectrum disorders. Behav Brain Res. 2015;278: 244-56.

20. Wischhof L, Irrsack E, Osorio C, Koch M. Prenatal LPS-exposure-a neurodevelopmental rat model of schizophrenia -differentially affects cognitive functions, myelination and parvalbumin expression in male and female offspring. Prog Neuropsychopharmacol Biol Psychiatry. 2015;57: 17-30. 21. Gabbita SP, Johnson MF, Kobritz N, Eslami P, Poteshkina A, Varadarajan S, Turman J, Zemlan F, Harris-White ME.2015. Oral TNFa modulation alters neutrophil infiltration, improves cognition and diminishes tau and amyloid pathology in the 3xTgAD mouse model. PLoS One. 2015;10: e0137305. 22. Kostrzewa RM. Dopamine receptor supersensitivity. Neurosci Biobehav Rev. 1995;19: 1-17.

23. Zhu F, Zheng Y, Liu Y, Zhang X, Zhao J. Minocycline alleviates behavioral deficits and inhibits microglial activation in the offspring of pregnant mice after administration of polyriboinosinicpolyribocytidilic acid. Psychiatry Res. 2014;219: 680-6.

24. Robbins TW. Neurobehavioural sequelae of social deprivation in rodents revisited: Modelling social adversity for developmental neuropsychiatric disorders. J Psychopharmacol. 2016;30:1082-1089.

25. Clark SM, Notarangelo FM, Li X, Chen S, Schwarcz R, Tonelli LH. Maternal immune activation in rats blunts brain cytokine and kynurenine pathway responses to a second immune challenge in early adulthood. Prog Neuropsychopharmacol Biol Psychiatry. 2018;89: 286-294.

26. Lee SM, Yune TY, Kim SJ, Kim YC, Oh YJ, Markelonis GJ, Oh TH. Minocycline inhibits apoptotic cell death via attenuation of TNFa expression following iNOS/NO induction by lipopolysaccharide in neuron/glia co-cultures. J Neurochem. 2004;91: 568-78. 27. Giovanoli S, Engler H, Engler A, Richetto J, Feldon J, Riva MA, Schedlowski M,Meyer U. Preventive effects of minocycline in a neurodevelopmental two-hit model with relevance to schizophrenia. Transl Psychiatry. 2016;6: e772. 28. Kolanowski A, Boltz M, Galik E, Gitlin LN, Kales HC, Resnick B, Van HaitsmaKS, Knehans A, Sutterlin JE, Sefcik JS, Liu W, Petrovsky DV, Massimo L,Gilmore-Bykovskyi A, MacAndrew M, Brewster G, Nalls V, Jao YL, Duffort $\mathrm{N}$, Scerpella D. Determinants of behavioral and psychological symptoms of dementia: A scoping review of the evidence. Nurs Outlook. 2017; 65:515-529.

29. Javitt DC, Freedman R. Sensory processing dysfunction in the personal experience and neuronal machinery of schizophrenia. Am J Psychiatry. 2015;172: 17-31.

30. Li L, Du Y, Li N, Wu X, Wu Y. Top-down modulation of prepulse inhibition of the startle reflex in humans and rats. Neurosci Biobehav Rev. 2009;33: 1157-67.

31. Flinkkilä E, Keski-Rahkonen A, Marttunen M, Raevuori A. Prenatal inflammation, infections and mental disorders. Psychopathology. 2016;49: 317-333. 
32. Meyer U. Prenatal poly $(\mathrm{I}: \mathrm{C})$ exposure and other developmental immune activation models in rodent systems. Biol Psychiatry. 2014;75: 307-15.33. Leviton A, Gressens P. Neuronal damage accompanies perinatal white-matter damage. Trends Neurosci. 2007;30: 473-8. 34. Khwaja 0, Volpe JJ. Pathogenesis of cerebral white matter injury of prematurity. Arch Dis Child Fetal Neonatal Ed. 2008;93: F153-61. 35. Osborne AL, Solowij N, Babic I, Huang XF, Weston-Green K. Improved social interaction, recognition and working memory with cannabidiol treatment in a prenatal infection (poly l:C) rat model. Neuropsychopharmacology. 2017;42: 1447-1457. 36. Ozawa K, Hashimoto K, Kishimoto T, Shimizu E, Ishikura $\mathrm{H}$, lyo $\mathrm{M}$. Immune activation during pregnancy in mice leads to dopaminergic hyperfunction and cognitive impairment in the offspring: a neurodevelopmental animal model of schizophrenia. Biol Psychiatry. 2006;59: 546-54.

37. Buschert J, Sakalem ME, Saffari R, Hohoff C, Rothermundt M, Arolt V, et al. Prenatal immune activation in mice blocks the effects of environmental enrichment on exploratory behavior and microglia density. Prog Neuropsychopharmacol Biol Psychiatry. 2016;67:10-20.

38. Meehan C, Harms L, Frost JD, Barreto R, Todd J, Schall U, Shannon Weickert C, Zavitsanou K, Michie PT, Hodgson DM. Effects of immune activation during early or late gestation on schizophrenia -related behavior in adult rat offspring. Brain Behav Immun. 2017;63: 8-20. 39. Piontkewitz Y, Bernstein HG, Dobrowolny H, Bogerts B, Weiner I, Keilhoff G. Effects of risperidone treatment in adolescence on hippocampal neurogenesis,parvalbumin expression, and vascularization following prenatal immune activation in rats. Brain Behav Immun. 2012; 26:353-63. 40. Murray KN, Edye ME, Manca M, Vernon AC, Oladipo JM, Fasolino V, Harte MK,Mason V, Grayson B, McHugh PC, Knuesel I, Prinssen EP, Hager R, Neill JC. Evolution of a maternal immune activation ( $\mathrm{mlA}$ ) model in rats: Early developmental effects. Brain Behav Immun. 2018;75:48-59.41. Smolders S, Notter T, Smolders SMT, Rigo JM, Brône B. Controversies and prospects about microglia in maternal immune activation models for neurodevelopmental disorders. Brain Behav Immun. 2018;73: 51-65.

42. Echeverria V, Yarkov A, Aliev, G. 2016. Positive modulators of the a7 nicotinic receptor against neuroinflammation and cognitive impairment in Alzheimer's disease. Prog Neurobiol. 2016;144: 14257.

43. Wan L, Thomas Z, Pisipati S, Jarvis SP, Boutros NN. Inhibitory deficits in prepulse inhibition, sensory gating, and antisaccade eye movement in schizotypy. Int J Psychophysiol. 2017;114: 47-54. 44. Potasiewicz A, Nikiforuk A, Hołuj M, Popik P. Stimulation of nicotinic acetylcholine alpha7 receptors rescue schizophrenia -like cognitive impairments in rats. J Psychopharmacol. 2017;31: 260-271.

45. Benros ME, Nielsen PR, Nordentoft M, Eaton WW, Dalton SO, Mortensen PB. Autoimmune diseases and severe infections as risk factors for schizophrenia: a 30-year population-based register study. Am J Psychiatry. 2011;168: 1303-10.

46. Bayer TA, Buslei R, Havas L, Falkai P. Evidence for activation of microglia in patients with psychiatric illnesses. Neurosci Lett. 1999;271:126-8. 
47. Monji A, Kato T, Kanba S. Cytokines and schizophrenia: microglia hypothesis of schizophrenia. Psychiatry Clin Neurosci. 2009;63: 257-65.

48. Monji A, Kato TA, Mizoguchi Y, Horikawa H, Seki Y, Kasai M, Yamauchi Y, YamadaS, Kanba S. Neuroinflammation in schizophrenia especially focused on the role of microglia. Prog Neuropsychopharmacol Biol Psychiatry. 2013;42:115-121. 49. Miller BJ, Buckley PF, McEvoy JP. Inflammation, substance use,psychopathology, and cognition in phase 1 of the clinical antipsychotic trials ofintervention effectiveness study. Schizophr Res. 2018;195:275-282.

50. De Picker LJ, Morrens M, Chance SA, Boche D. Microglia and brain plasticity in acute psychosis and schizophrenia illness course: A meta-review. Front Psychiatry. 2017;8: 238.

51. Sommer IE, de Witte L, Begemann M, Kahn RS. Nonsteroidal anti-inflammatory drugs in schizophrenia: ready for practice or a good start? A meta-analysis. J Clin Psychiatry. 2012;73: 414-9.

52. Solmi M, Veronese N, Thapa N, Facchini S, Stubbs B, Fornaro M, Caravalho AF, Correll CU. Systematic review and meta-analysis of the efficacy and safety of minocycline in schizophrenia. CNS Spectr. 2017;22: 415-426.

53. Xiang YQ, Zheng W, Wang SB, Yang XH, Cai DB, Ng CH, Ungvari GS, Kelly DL, Xu WY, Xiang YT. Adjunctive minocycline for schizophrenia: A meta-analysis of randomized controlled trials. Eur Neuropsychopharmacol. 2017;27: 8-18.

54. Kusumi I, Boku S, Takahashi Y. Psychopharmacology of atypical antipsychotic drugs: From the receptor binding profile to neuroprotection and neurogenesis. Psychiatry Clin Neurosci. 2015;69: 243-58.

55. Hunter A, Murray R, Asher L, Leonardi-Bee, J. The effects of tobacco smoking, and prenatal tobacco smoke exposure, on risk of schizophrenia: a systematic review and meta-analysis. Nicotine Tob Res. 2018;

56. Featherstone RE, Siegel SJ. The role of nicotine in schizophrenia. Int Rev Neurobiol. 2015;124 23-78.

\section{Abbreviations}

SCHZ: Schizophrenia; TNFa: Tumor Necrosis Factor Alpha; Poly I:C: Polyinosinic:polycytidylic acid; NQ: neonatal quinpirole treatment; NS: neonatal saline treatment. PPI: Prepulse inhibition

\section{Declarations}

Ethical approval and consent to participate: Not applicable

\section{Consent for publication: Not applicable}


Availability of data and materials: The datasets used and/or analyzed during the current study are available from the corresponding author on reasonable request

Competing interests: SPG declares a financial competing interest. All other authors declare no competing interests.

Funding: Provided by a Graduate Student grant from the ETSU School of Graduate Studies to Heath W. Shelton and P2D Bioscience, Inc.

\section{Authors' Contributions:}

Heath W. Shelton contributed to writing of the manuscript and was involved in every aspect of the project; Somasundar Prasad Gabbita contributed to writing of the manuscript and is the Chief Executive Officer (CEO) of P2D Bioscience, Inc., in Cincinnati, OH, and designed the TNFa modulator PD2024;

W. Drew Gill was primarily involved in behavioral testing of the animals and data analysis;

Wyatt S. Whicker was primarily involved in behavioral testing of the animals;

Katherine C. Burgess was primarily involved in behavioral testing of the animals and data analysis, as well as some management of the scheduling of behavioral testing and software management;

Russell W. Brown was involved in writing of the manuscript, data analysis, and management of the entire project. 